\title{
QANATES, CIMBRAS Y MINADOS EN EL VALLE DEL ALMANZORA (SURESTE DE ESPAÑA) ${ }^{1}$
}

\author{
José María Gómez Espín \\ Departamento de Geografía. Universidad de Murcia.
}

\section{RESUMEN}

Los sistemas de captación de freáticos próximos a la superficie, mediante la técnica del pozo horizontal cubierto (qanates, cimbras y minados), están muy extendidos en medios semiáridos como el Valle del Almanzora. En Octubre de 2001, la Junta Central de Usuarios de Aguas del Valle del Almanzora, en el inventario de comunidades de regantes, reflejaba como recursos propios la captación de agua por 4 cimbras, 75 pozos y 1 desaladora en el Bajo Almanzora; de 12 pozos, 7 cimbras y 8 fuentes en el Medio Almanzora; y de 9 pozos, 2 cimbras y 8 fuentes en el Alto Almanzora. Estos recursos propios de las cimbras habían mantenido unos espacios de regadío tradicional, con variaciones significativas en los volúmenes captados según la sucesión de lluvias y sequías. En los últimos años asegurar los abastecimientos y la ampliación de los espacios regados ha sido posible gracias a la apertura de multitud de pozos, a la traída de caudales foráneos (Trasvase Tajo-Segura y Conexión Negratín-Almanzora), y a la puesta en marcha de varias desaladoras. El objeto de este trabajo es descubrir y explicar el estado de la relación oferta de recursos-demandas de agua, desde el análisis geográfico regional de un espacio como el Valle del Almanzora en el Sureste de España.

Palabras clave: Pozo horizontal cubierto; qanat; cimbra; minado; río-rambla; medio semiárido; Sureste España.

1. Proyecto 17.587. «Eficiencia y sostenibilidad en el uso del agua en las zonas regables del Trasvase Negratín-Almanzora: Modernización de regadíos». Agradecimiento a Enrique Fernández Bolea, a Lorenzo López Asensio, y Encarnación Gil Meseguer por la documentación y fotografías cedidas. Y a los gestores de agua Matías Gómez. Fernando Haro, José Miras, Prudencia López, Blas Jiménez, José Fuentes, Luis Soler, Pedro Chacón, Antonio Quiles, Angel Torrecilla, etc., por la información suministrada para la mejora de este trabajo. 


\section{ABSTRACT}

Qanates, cimbres and mined in the valley of the Almanzora (Southeast of Spain)

The reception systems of phreatic close to the surface, using the technique of the covered horizontal well (qanates, cimbres and mined), are really extended in semiarid means like the Vale of the Almanzora. In October, 2001, the Central Meeting of water Users of the Vale of the Almanzora, in the inventory of communities of resackcloths, was reflecting like own funds the water reception for 4 cimbras, 75 wells and 1 desalination plant in Low Almanzora; of 12 wells, 7 cimbras and 8 sources in the Way Almanzora; and of 9 wells, 2 cimbras and 8 sources in the High place Almanzora. These own funds of the cimbras had maintained a few spaces of traditional irrigation, with significant changes in the volumes received according to the sequency of rains and droughts. In the last years, to make sure the supplyings and the enlargement of the watered spaces, it has been possible thanks to the opening of multitude wells and the water brought from foreign water flows (I transferred Tagus - Segura and Connection Negratín-Almanzora), as well as to the starting of several desalination plants. The object of this work is to discover and to explain the state of the relation offers of water resources - demands, from the regional geographical analysis of a space like the Vale of the Almanzora in the Southeast of Spain.

Keywords: Covered horizontal well; qanat, cimbre, mined gallery, river-boulevard; semiarid area; South-east of Spain.

\section{LA TÉCNICA DEL POZO HORIZONTAL CUBIERTO (GALERÍA), UNA FORMA DE} ALUMBRAR AGUA EN MEDIOS SEMIÁRIDOS COMO EL VALLE DEL ALMANZORA (SURESTE DE ESPAÑA)

El Valle del Almanzora, es una depresión sinclinal alargada en sentido OesteEste. Enmarcada por los relieves de la Sierra de Las Estancias al Norte, y por los relieves de la Sierra de Los Filabres al Sur. Los tramos alto y medio responden a esa orientación general en sentido de los paralelos; pero en su tramo bajo se encuentra con el relieve de la Sierra de Almagro y cambia a una dirección NW-SE, casi meridiana, para desembocar en el Mediterráneo, cerca de Villaricos. De las cumbres de Las Estancias (1.467 metros s.n.m.) y de los Filabres (2.168 metros s.n.m.) descienden superficies de glacis de acumulación (hasta cinco niveles con pendiente hacia el talweg), cuyas escorrentías recoge el Almanzora y su red afluente.

Los materiales de estos piedemontes (glacis y glacis-cono), facilitan la infiltración de las aguas de lluvia, así como las del deshielo de la nieve acumulada en las cumbres; parte de estas aguas son captadas mediante pozos horizontales drenantes, excavados, del tipo qanat (galería con lumbreras y pozo madre, en ocasiones identificado como fuente.). En los cursos del tipo rambla o rio-rambla como el Almanzora y la mayor parte de su red afluente, existen en lugares del 
lecho «alveos» (vasos de embalse subterráneo) de gran interés hidrogeológico en un entorno de escasez hídrica, como el Sureste de la Península Ibérica, región natural de la que forma parte la Cuenca del Almanzora. Esta circulación subsuperficial, entre los intersticios que dejan gravas y arenas, es alumbrada mediante pozos horizontales que se abren en estos sedimentos para construir galerías filtrantes del tipo tajea o cimbra, y alumbrar las aguas que se han introducido

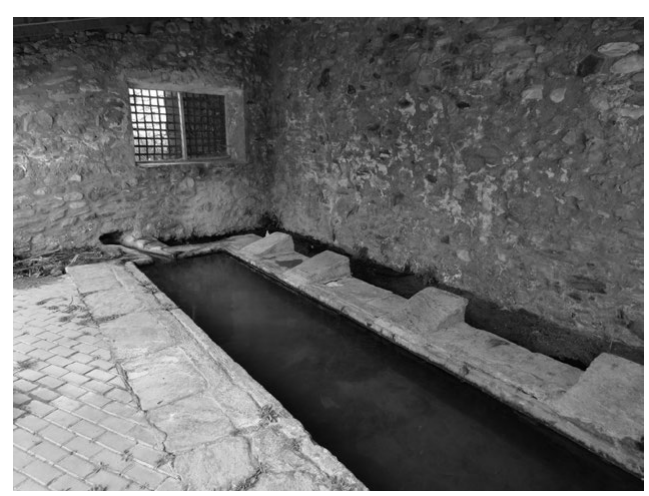

Figura 1. Lavadero con las aguas alumbradas por el Cañico de Olula del Río.

Fuente: Gómez Espín, J.M. a 14/03/2015. por las cubiertas y paredes de estas galerías.

El ámbito territorial de la Junta Central de Usuarios de Aguas del Valle de Almanzora, (constituida el 29 de noviembre de 1987, y aprobados sus estatutos por Resolución de la Confederación Hidrográfica del Sur el 9 de agosto de 1988), está subdividido en tres zonas y definido por los siguientes términos municipales. Zona Alta: municipios de Alcóntar, Serón, Bayarque, Tíjola, Armuña, Lucar, Somontín, Sierro, Suflí, Urracal y Purchena. Zona Media: municipios de Olula del Río, Macael, Fines, Cantoria, Partaloa, Oria, Albox, Arboleas, y Taberno. Zona Baja: municipios de Zurgena, Huércal-Overa, Antas, Vera, Pulpí, y Cuevas del Almanzora.

El objeto de este estudio es descubrir y explicar aquellos recursos propios de agua en el Valle del Almanzora que son alumbrados con técnicas de pozo horizontal cubierto. El papel que han tenido y tienen a nivel local para el abastecimiento de personas y ganados, para usos domésticos e industriales de los vecinos, y para el mosaico de huertas. Metodológicamente es un estudio regional, que desde el análisis, diagnóstico y prognosis territorial permite valorar la participación de estas aguas alumbradas en la relación recursos-demandas de años hidrológicos como 2014/2015. El trabajo de campo, el análisis de la

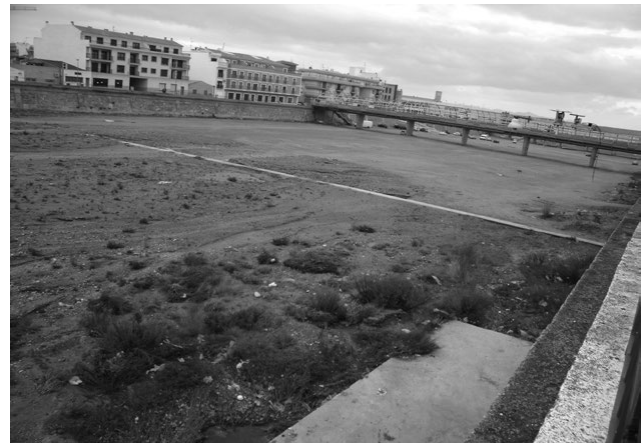

Figura 2. El río Almanzora, encauzado a su paso por Cuevas de Almanzora.

Fuente: Gómez Espín, José M.a 10/02/2015. 
documentación de diferentes archivos, y las entrevistas con gestores del agua ha permitido conocer este patrimonio hidráulico (molinos, almazaras, lavaderos, abrevaderos, bocaminas, lumbreras, canalizaciones, etc.,), la funcionalidad o no de las técnicas del pozo horizontal cubierto (qanates, cimbras, y minados), y la gestión social del agua en algunos de estos sistemas.

ReCursos De Fuentes y Cimbras EN EL VAlle Alto y Medio Del Almanzora (ALMERÍA)

En el Inventario de Comunidades de Regantes de la Comarca del Almanzora (octubre, 2001), sobresalen entre las comunidades de regantes de la Zona Alta, las de: Fuente de la Zanja de Sufli, Zanja de Chaulena y Padules, Zanja de Gadil, Fuengrande de los Cortijillos, Fuente de Cela, Fuente Vieja, Nuestra Señora del Socorro, Palomino, San Antonio de El Higueral, San Pedro, San Rafael, de Tíjola, Fuente de Las Parras, de la Vega de Serón, Cimbra de la Lágrima-Pago Angosto. La mayor parte de los caudales para riego corresponden a recursos propios de fuentes y zanjas o tajeas, también a los de pozos y sondeos. Así la Zanja de Sufli, conocida como la cimbra de la Zanja en el lecho del Almanzora, en el 2001, se aforó en 25 litros por segundo para riego de unas 27 ha, en verano el caudal baja hasta $0,5 \mathrm{l} / \mathrm{seg}$. La Zanja de Chaulena y Padules, también es una cimbra que capta la circulación subálvea en el lecho del Almanzora, de un caudal estimado de 15 1/seg., en situaciones de sequía, como en 1988, puede secarse. La Fuente de Cela, es un nacimiento que surge del fondo de una balsa entre los términos de Lúcar y Tíjola (los vecinos del lugar dicen que no sufre variaciones el caudal de unos 43 1/seg., hablan de un río subterráneo que viene de Sierra Nevada); riega más de 100 ha. La cimbra de La Lágrima, tenía un aforo estimado, en el 2001, de 50 1/seg., y regaba 16 ha.

En la Zona del Medio Almanzora sobresalen como sistemas de alumbrar

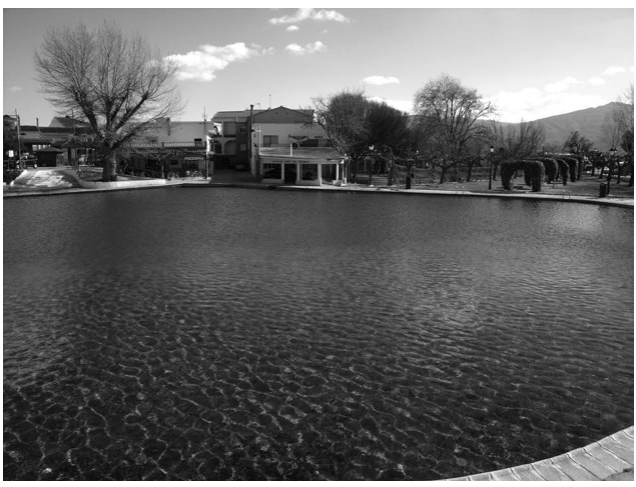

Figura 3. Balsa del nacimiento de la Fuente de Cela. Fuente: Gil Meseguer, E. 21/01/2015. recursos propios el qanat de la Fuente del Marqués, en Albox; la cimbra de la Fuente del Beneficiado de Fines y el Cañico de Olula del Río, y varias galerías abiertas en el lecho del Almanzora como la cimbra de San Mateo en la C.R. Arroyo Aceituno, en Arboleas. Esta última, en el estudio inventario de 2001, figura con un caudal estimado de $12 \mathrm{l} / \mathrm{seg}$, superior al de la cimbra de la Cueva con 7 1/ seg., la del Molino con 3 1/seg., y 
sin caudal alumbrado (seca) la de la Alquería,

La galería del Cañico de Olula del Río, de caudal escaso y variable, cuenta con la Fuente de los Caños, (desde 1867, realizada por suscripción de los vecinos), abrevadero y lavadero. Se une a la cimbra de la Fuente del Beneficiado para regar en Olula del Río el paraje de «El Margen» (24 horas de sábado a domingo) y otros pagos de Fines.

La cimbra de la Fuente del Beneficiado, cuenta con más de trescientos metros de galería en esa margen izquierda del Almanzora. En la captación de caudales entre las arenas y gravas del cauce la circulación subsuperficial aumenta con el deshielo de nieves en Bacares, con caudal superior a los 30 1/seg., que disminuye en las situaciones de sequía a apenas $5 \mathrm{l} /$ seg. Las aguas alumbradas se distribuyen en dos tandas (de día y de noche). En la de noche (de 20 horas de la tarde a 8 horas de la mañana) las aguas se acumulan en una balsa de 45 metros de larga, 27 metros de ancha, y unos 3 metros de profundidad, con una capacidad máxima de $3.645 \mathrm{~m}^{3}$. A partir de las 8 de la mañana hay dos chorros para riego, las aguas alumbradas directamente de la bocamina que riegan los pagos más altos; y las aguas que salen de la balsa. El espacio regable de unas 87 ha., cuenta con la ayuda de caudales subterráneos del Pozo Collado Puches, ya que la cimbra a primeros de Enero de

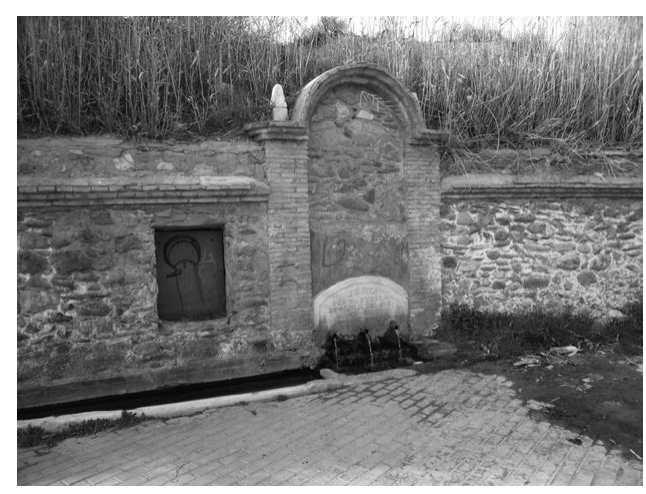

Figura 4. Fuente de los Caños y abrevadero con las aguas alumbradas por la galería del Cañico de Olula del Río. Fuente: Gómez Espín, J.M. ${ }^{a}$ $13 / 03 / 2015$.

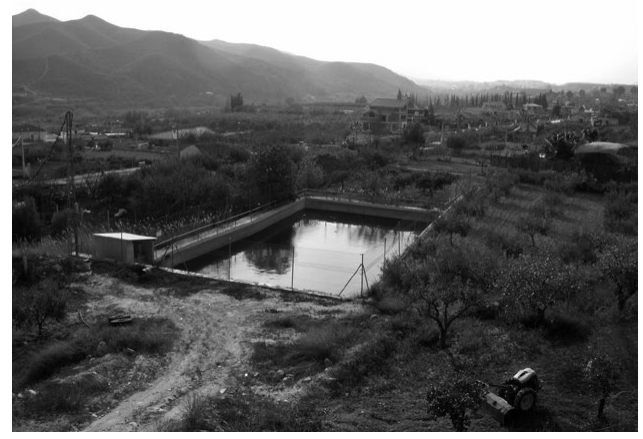

Figura 5. Balsa que recibe las aguas alumbradas por la cimbra Fuente del Beneficiado de Fines.

Fuente: Gómez Espín, J.M. ${ }^{a}$ 13/03/2015.

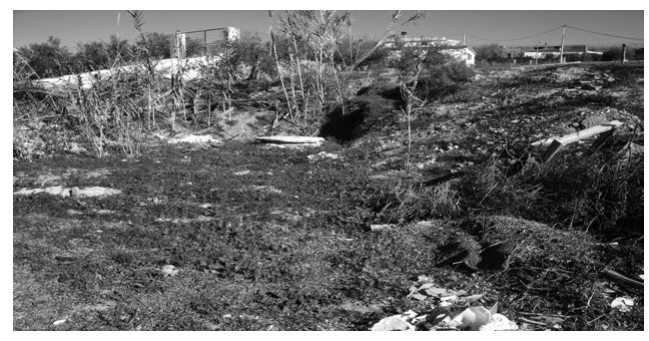

Figura 6. Última lumbrera del qanat de la Fuente del Marqués (Albox). Fuente: Gil Meseguer, E. $04 / 01 / 2015$. 
2015 alumbraba sólo un caudal de 12,4 1/ seg. Las tierras regadas se orientan a olivar, a cítricos y a hortícolas. Una parte de ellas ya se riegan a presión (riego localizado) tras un proceso de modernización de regadíos que ha llevado a cabo la comunidad de regantes.

El qanat de la Fuente del Marqués, en Albox, arranca de un pozo madre (a 28 metros de profundidad entre las arenas brotan las aguas) en la margen izquierda de la rambla de Albox, en el paraje de Locaiba. Cuenta con tres lumbreras antes de atravesar la rambla para regar en la otra margen el paraje de Ruiz, y de nuevo atraviesa en diagonal el lecho de la rambla hasta el paraje de Aljara, dónde todavía se observa la última lumbrera, la bocamina, el partidor, las conducciones para riego y los restos de cuatro molinos hidráulicos de cubo movidos por estas aguas alumbradas en esa margen izquierda..

Galerías en el Bajo Almanzora (Zurgena, Vera, Antas, HuércalOvera, Cuevas de Almanzora, Pulpí)

En Zurgena como aprovechamientos tradicionales de agua alumbrada mediante minados hemos de señalar: La Minica, la Fuente de la Capellania, y la Fuente del Peral. La galería de la Minica, está excavada en el margen izquierdo del Almanzora, en el lugar de El Alto paraje de «La Alfoquía», cuenta con unos 120 metros de longitud, una altura de galería de 1,40 metros, y con dos lumbreras. La salida de la bocamina desemboca en una pequeña balsa, donde se acumula el agua alumbrada para el riego de cítricos, a pesar de ser un poco salada.

La Fuente de la Capellanía es un minado en lal margen derecha de la rambla de la Higuera, que antiguamente con su pequeño caudal entandado regaba varias huertas de cítricos, (en Abril del 2015 estaba seca).

La Fuente de los Llanos del Peral es un minado en la margen izquierda de la rambla del Peral, apoyado sobre

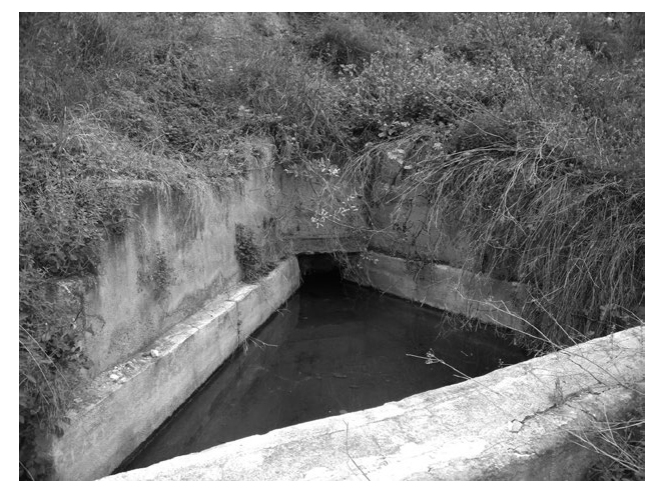

Figura 7. Bocamina y balsa de La Minica. (Paraje de la Alfoquía en Zurgena).

Fuente: Gil Meseguer, E. 06/04/2015. un conglomerado de la rambla. En Abril del 2015 apenas suministraba unos hilillos de agua a través de dos caños para abastecimiento de personas (rehabilitado el espacio como merendero), otro caño para el abrevadero de ganado (aguadero concejil junto a vereda de ganado), y finalmente el caudal sobrante se destinaba a riego entandado de varias huertas de cítricos.

La comunidad de regantes de la Fuente de Capellanía y Llanos 
del Peral, en la actualidad, basa su principal recurso de agua en caudales externos de la Conexión Negratín-Almanzora, para su área regable de de más de 220 ha, dedicadas a cítricos, pero dónde algún agricultor está experimentando con frutales como caqui y membrillo.

El artículo 2 de los estatutos de la «Comunidad de Regantes de San Ramón Nonnato» de Zurgena, dice así: «Pertenecen a la Comunidad: las galerías, boqueras, tomas y cauces en el Río Almanzora, Rambla

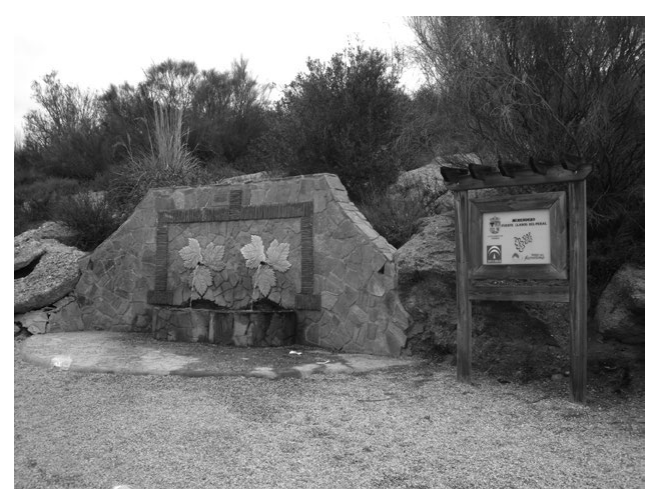

Figura 8. Fuente de los Llanos del Peral. (Zurgena). Fuente: Gil Meseguer, E. 06/04/2015. del Peral y Almalejo en los términos municipales de Zurgena y Arboleas...Y obras correspondientes a las tomas y conducciones del Trasvase del Negratín al Almanzora, según Resolución de 3 de agosto de 2004».

Tabla 1. Aprovechamientos para riego que la C.R. «San Ramón Nonnato» tiene de las aguas procedentes del río Almanzora y de fuentes

\begin{tabular}{|l|c|c|l|}
\hline \multicolumn{1}{|c|}{ Denominación } & $\begin{array}{c}\text { Caudal inscrito } \\
\text { l/seg. }\end{array}$ & $\begin{array}{c}\text { Superficie de } \\
\text { riego Ha. }\end{array}$ & $\begin{array}{c}\text { Término } \\
\text { municipal }\end{array}$ \\
\hline Cimbra Alfoquía & 5,52 & 24,8776 & Arboleas \\
\hline Cimbra Molino o Matorno & 12,80 & 57,6547 & Arboleas \\
\hline Cimbra Calafa & 5,63 & 25,3761 & Zurgena \\
\hline Galería y toma Palaces & 12,61 & 56,8274 & Zurgena \\
\hline Toma Tablares & 4,36 & 19,6375 & Zurgena \\
\hline Galería de Las Peñicas & 0,95 & 4,2670 & Zurgena \\
\hline Cimbra de Tamarchete & 18,56 & 83,6340 & Zurgena \\
\hline TOTALES & 60,43 & 272,2743 & Arb, Zurg. \\
\hline
\end{tabular}

Fuente: Elaboración propia. Modificación parcial de los Estatutos de la «Comunidad de Regantes San Ramón Nonnato» de Zurgena (Almería). Resolución de 25 de octubre de 2015.

También en Zurgena, varias sociedades agrarias de transformación (S.A.T.), contaban entre sus recursos propios con los alumbrados por cimbras. Así la S.A.T n. ${ }^{\circ} 309$ «Ahorrativo y Descanso de Paleces de Zurgena» figuraba en el Inventario de Comunidades de Regantes de la Comarca del Almanzora, del año 2001, con una cimbra de caudal variable. La S.A.T n. ${ }^{\circ} 7.255$ de Zurgena figuraba con tres cimbras de caudal variable según la estación del año. 
En Huércal Overa son numerosas las fuentes alumbradas por minados, sobresale en el paraje de Gacia, el qanat de la Virgen de los Desamparados; y en el de Los Gibaos (Cabezo de la Jara), el qanat de la Sociedad Colectiva de Aguas de la Asunción o galería del Cabezo. (Gil, E y Gómez, J.M. ${ }^{a}$ 2014). En este trabajo estudiamos, un minado de escaso aforo como la Fuente de Las Perdices, en Nieva; y una galería más compleja, como el qanat de la Fuente de Las Norias o de Nuestra Señora del Rosario.

Tabla 2. Estructura de la propiedad, según tiempos, en la tanda de la Fuente de las Perdices. (Huércal-Overa). (Tanda de 14 días)

\begin{tabular}{|c|c|c|c|}
\hline Día & Nombre y apellidos & Horas & Minutos \\
\hline $1,2,3$ & Pedro Benítez & 65 & 33 \\
\hline $3,10,11,14$ & José Parra Sánchez & 11 & 0 \\
\hline 3,4 & Juan García Sánchez & 26 & 39 \\
\hline 4,5 & Alfonso Vilar & 33 & 60 \\
\hline 5,6 & Ángel Martínez & 17 & 5 \\
\hline 6 & Matías Ballesta & 6 & 16 \\
\hline 6 & Juan García & 2 & 0 \\
\hline 6,7 & Elisa García & 1 & 40 \\
\hline 7 & Lucrecia Cerezuela & 1 & 40 \\
\hline 7 & Blas Sánchez & 13 & 10 \\
\hline 7 & José Viúdes & 8 & 44 \\
\hline 7,8 & Bartolomé Sánchez & 13 & 0 \\
\hline 8,9 & Venancio Navarro & 14 & 0 \\
\hline 9 & Diego García & 12 & 20 \\
\hline 9,10 & Teresa García Gilaber & 21 & 0 \\
\hline 10 & María Asunción Gilaber & 9 & 15 \\
\hline 11 & José Gómez & 11 & 0 \\
\hline 11 & Francisco García & 5 & 54 \\
\hline 11 & Baltasar Parra Sánchez & 4 & 40 \\
\hline 11,12 & José García & 13 & 56 \\
\hline 12 & Juan García & 10 & 28 \\
\hline 12,13 & Bartolomé Zurano & 16 & 18 \\
\hline 13 & Juan de Águeda & 2 & 0 \\
\hline 13 & Rosa García & 6 & 0 \\
\hline 13,14 & Francisco Bonillo & 13 & 22 \\
\hline 14 & José Benítez & 5 & 0 \\
\hline Subtotales & & 325 & 660 \\
\hline TOTAL & & 336 & \\
\hline
\end{tabular}

Fuente: Elaboración propia. Tandeo de la Fuente de las Perdices, en el año 1942. 
La Fuente de las Perdices, en Nieva, es un minado pequeño, de tipo qanat, con la bocamina en la misma balsa en la que se acumulan las aguas, según horas de cada propietario, en tanda de 14 días. (Tabla 2). Forma parte de los recursos propios de la C.R. «Zona Norte de Huércal Overa». Riega sobre todo olivar, al que algunos propietarios aplican riego localizado por «goteo».

La galería de la Fuente de Las Norias o de Nuestra Señora del Rosario, alumbraba agua para abastecimiento de personas y ganados en Las Norias (HuércalOvera). Cuenta con cuatro lumbreras y a la bocamina disponía también de lavadero para los usos domésticos de esta pedanía, que en la actualidad ha sido enterrado. En origen, quizás contaba la galería con más lumbreras hacia el piedemonte (glacis) de la Sierra de Enmedio, pero algunas de ellas han sido ocultadas (rellenadas y tapadas) para la ordenación de la nueva agricultura de horticultura inten-

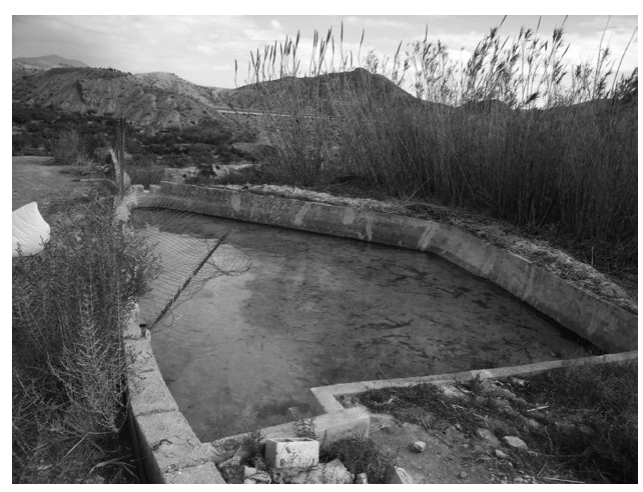

Figura 9. Balsa de la Fuente de las Perdices, en Nieva (Huércal-Overa).

Fuente: Gómez Espín, J.M. ${ }^{a}$ 19/02/2015.

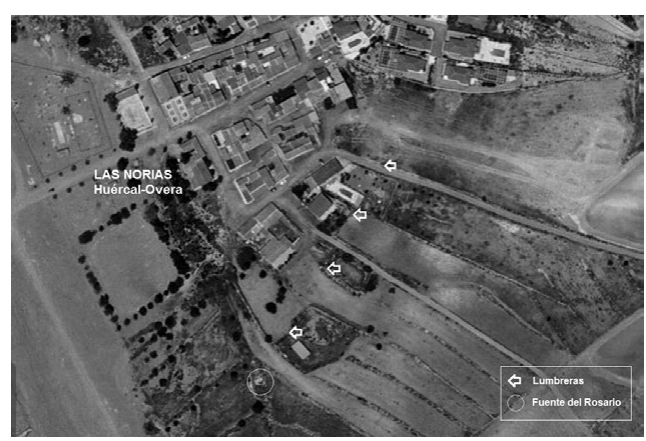

Figura 10. Galería de la Fuente de Las Norias o de Nuestra Señora del Rosario, en la pedanía de Las Norias (Huércal-Overa). Fuente: López Asensio, L. 27/04/2015. siva, que dispone como recursos de las aguas subterráneas profundas y de las de la Conexión Negratín-Almanzora.

En el Bajo Almanzora, en el lecho del río cercano a la Población de Cuevas, conviene mencionar los trabajos de alumbramiento de agua de la Cimbra del Cebollar o Mina de Toribio, que en la actualidad ha quedado bajo el vaso del embalse de la Presa de Cuevas, en el paraje del Peñón de Panes. Los trabajos para su construcción se prolongaron en el tiempo más de siglo y medio, de 1719 a 1878. Además de Toribio Martínez de la Vega, que la inicia con la excavación de una zanja en el Peñón de Panes, a la altura del pago de El Cebollar; intervinieron otros técnicos como Idelfonso Cerdá, Juan Fernando Feigenspan y Antonio de Falcés Yesares. La galería se prolongaba unos tres kilómetros desde el paraje del Peñón de Panes hasta el paraje de la Cueva o Piedra Negra. En 


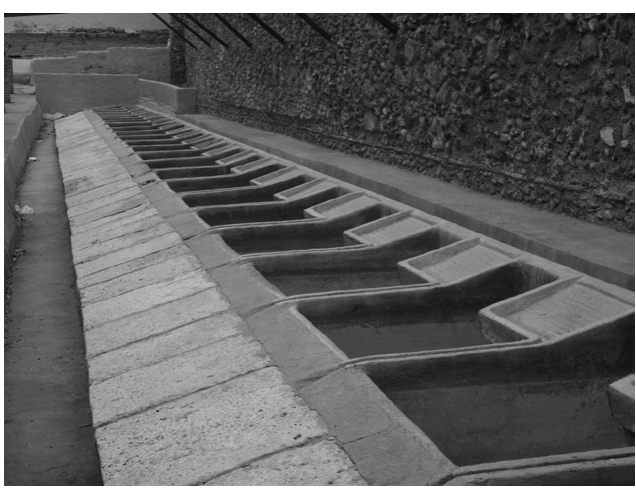

Figura 11. Lavadero público del Calvario, diseñado por los hermanos Siret, (Cuevas del Almanzora). Fuente: Gil Meseguer, E. 26/11/2009.

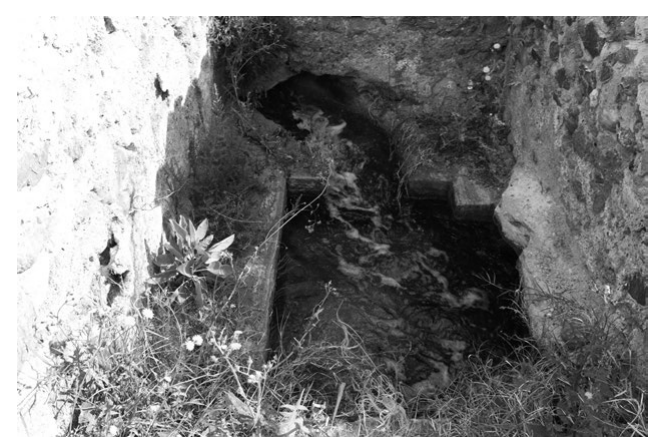

Figura 12. Arca o pilar, donde se parten las aguas alumbradas por la cimbra del Rincón o Atenores, en Guazamara (Cuevas del Almanzora). Fuente Gómez Espín, J, M. ${ }^{\text {a } 16 / 03 / 2015 . ~}$

su recorrido contaba con 47 lumbreras de 1,25 metros de diámetro. Las dimensiones eran de 1,80 metros de alto por 1,25 metros de ancho, con seis espacios para los descansos de los trabajadores y para almacenar materiales y herramientas. La bocamina vertía aguas sobre la parte superior del cauce de la margen derecha, uniéndose allí con las provenientes de la Fuente de Overa. (Fernández, E. 2006:142).

En Cuevas, la galería de mayor interés era la que le proporcionaría aguas para abastecimiento y usos domésticos. Traída el agua de la Ballabona (de la finca de D. José López Martínez), y en la que participaron los hermanos Siret. Enrique Siret, autor, junto a Antonio Petre, del Proyecto de traída de las aguas de la Ballabona para el Abasto de la Ciudad de Cuevas 1879. Luis Siret se ocuparía junto a su hermano de la dirección de las obras del acueducto y posteriormente de los trabajos de construcción de los depósitos. (Fernández, E. 2006: 268-269).

Al noreste del término de Cuevas del Almanzora, entre el borde suroriental de la Sierra de Almagro y los relieves litorales del Castillarico, Los Pinos y El Aguilón, se extiende el corredor de Guazamara, drenado por la rambla del mismo nombre que desagua en el Almanzora a través de la rambla de Canalejas. En la margen izquierda de la rambla de Guazamara se alumbraban aguas subsuperficiales a través de una cimbra de más de 300 metros, conocida como del Rincón o Atenores. En 1814 Miguel Soler Molina, acometió labores en busca del ansiado líquido dentro de los límites de su finca y muy cerca de la cimbra, por entonces seca, que construyeron los interesados del Rincón. Al percatarse del desarrollo de los trabajos, éstos se alarmaron porque temieron que cuando volviesen las lluvias las aguas que por allí corrieran serían recogidas por el nuevo cauce que 
se abría en mejores condiciones que el antiguo. A pesar de los vecinos, el hacendado creyéndose con pleno derecho, continuo impasible con las tareas iniciadas y ese tesón fue recompensado con el hallazgo de un venero que discurría entre las arenas de la rambla sin llegar a canalizarse por la vieja cimbra del Rincón. (Fernández, E. 2011: 38).

Las aguas de la nueva cimbra, en la margen izquierda de la rambla, y en el pago del Llano de los Rodríguez; por acuerdo entre los propietarios del sistema antiguo y de Miguel Soler Molina (documento de 14 de abril de 1815) serían utilizadas por mitad entre ambas partes. Se calculó en 48 1/ seg., para el común del Rincón y otro volumen semejante correspondería a Miguel Soler Molina para atender sus tierras en el Cortijo del Aire. Estos caudales alumbrados se partían en el arca o pilar, y eran conducidos a balsas dónde acumularlas para riego $\mathrm{y}$ otros usos. Así una parte se depositaban en la balsa del Rincón y la

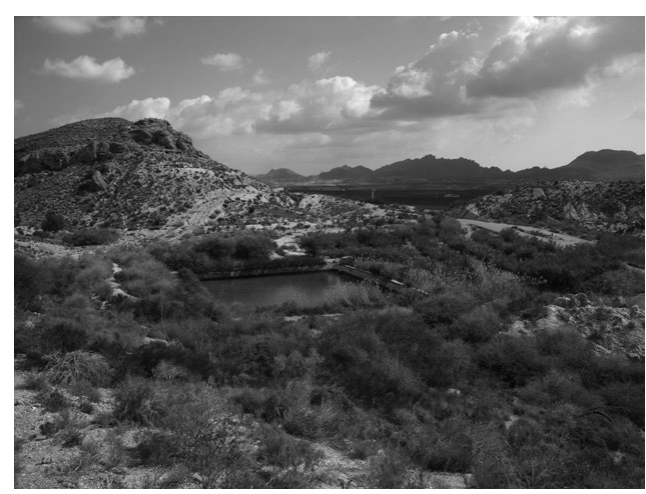

Figura 13. Balsa de Las Minas o del Molino, en Guazamara (Cuevas del Almanzora). Fuente: Gómez Espín, J.M. ${ }^{a}$ 16/03/2015

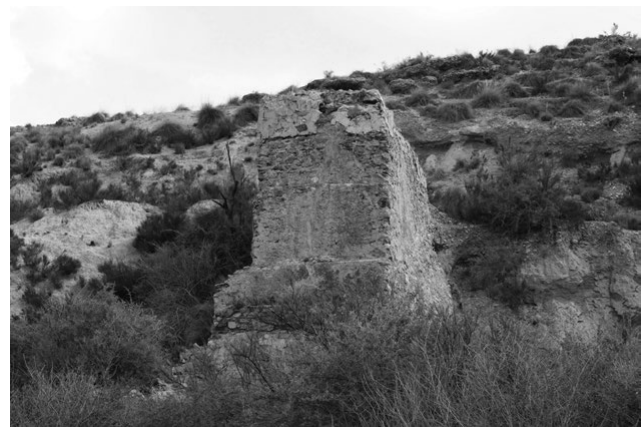

Figura 14 Restos del cubo del molino hidráulico construido por Miguel Soler Molina, en 1831. Guazamara (Cuevas del Almanzora). Fuente: Gómez Espín, J.M. a 04/04/2015. parte de Miguel Soler Molina, (tras regar las tierras del Cortijo del Aire), en la balsa de Las Minas o del Molino. Ésta era llamada así por recibir también las aguas de la galería de las Fuente de las Minas, que arrancaba de la Loma de Farax, con varias lumbreras en su recorrido. Con las aguas acumuladas en la balsa se regaban los pagos más bajos, y proporcionaban la energía a fábricas hidráulicas, como el molino hidráulico de cubo construido por Miguel Soler Molina en 1831 y, a la cercana almazara del Cortijo Colorao.

En este sector más oriental del término de Cuevas del Almanzora, se contaba en 1933 con las aguas de varias fuentes y manantiales: manantial del Taharal, del Rincón o de los Atenores, de las Canalejas, de las Gachas, de las Fuentecicas, de Malagón, los de Vizcaino. Algunos de ellos, en los últimos años, todavía 


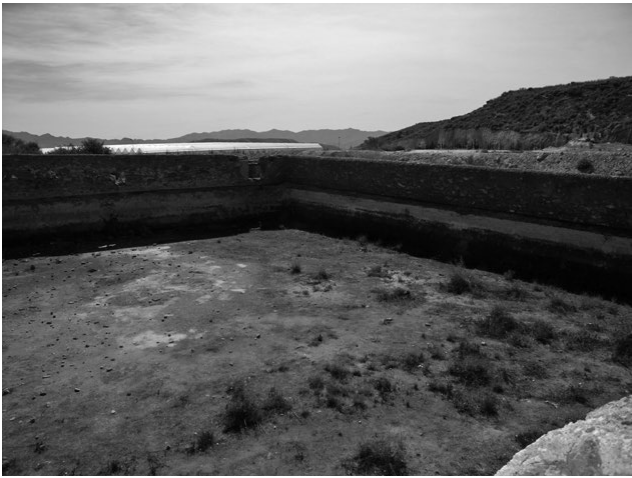

Figura 15. Restos de la Balsa de Antonio Falces, en la margen izquierda de la rambla Cano o de la Higuera (Los Guiraos). Fuente: Gómez Espín, J. M. ${ }^{\text {a } 30 / 03 / 2015 . ~}$

alumbran aguas como la cimbra del Rincón o Atenores y otros han quedado secos con la apertura de pozos en los años sesenta por el Instituto Nacional de Colonización y Desarrollo Rural. Así la apertura en Los Guiraos de los sondeos n. ${ }^{\circ} 1$ (en 1961) y n.o 2 (en 1962) secaron el alumbramiento que mediante galería de 25 metros se venía practicando en el barranco o cañada de Águila conocido como de Malagón. Y también disminuyó el aforo del qanat de la margen derecha de la rambla Cano (apenas $7 \mathrm{l} / \mathrm{seg}$.), cuyas aguas alumbradas atravesaban la rambla, mediante sifón, hasta la llamada balsa de Antonio Falces, que permitía el riego de tierras por encima de la Loma de Los Guiraos. Las protestas de todos estos usuarios llevaron al Instituto de Reforma y Desarrollo Agrario, en 1972, a un acuerdo de permutar derechos de agua. De forma gratuita, cada quince días, suministrarían 10 horas de agua del pozo n. ${ }^{\circ} 1(75 \mathrm{l} / \mathrm{seg}$. a Malagón y 32 horas a los de la Balsa de Falces. Más de las tres cuartas partes de los propietarios $(78,12 \%)$ de la Balsa de Antonio Falces reunían algo más de una cuarta parte $(30,17 \%)$ de las 360 horas de agua de la tanda de quince días. (Tabla 3).

Tabla 3. Estructura de la propiedad del agua de la Balsa de Antonio Falces en Los Guiraos. (Cuevas de Almanzora) (Tanda de 15 días)

\begin{tabular}{|l|c|c|l|c|}
\hline \multicolumn{1}{|c|}{ Escala } & N. $^{\mathbf{0}}$ propietarios & \% Propietarios & \multicolumn{1}{c|}{ Tiempo } & \% Tiempo \\
\hline De 0 a 3horas & 12 & 37,50 & $21 \mathrm{~h} 10 \mathrm{~min}$ & 5,86 \\
\hline De más de 3h a 10h & 13 & 40,62 & $87 \mathrm{~h} 39 \mathrm{~min}$ & 24,31 \\
\hline De más de 10h a 30h & 5 & 15,62 & $91 \mathrm{~h} 37 \mathrm{~min}$ & 25,43 \\
\hline De más de 30h a 60h & 1 & 3,13 & $48 \mathrm{~h} 0 \mathrm{~min}$ & 13,40 \\
\hline Más de 60h a 120h & 1 & 3,13 & $111 \mathrm{~h} \mathrm{34min}$ & 31,00 \\
\hline TOTALES & 32 & 100,00 & $360 \mathrm{~h}$ & 100,00 \\
\hline
\end{tabular}

Fuente: Elaboración propia. MINISTERIO DE AGRICULTURA. IRYDA. Relación de propietarios con derecho a agua gratuita y horas que le corresponden del Sondeo $n .^{\circ} 1$ de Los Guiraos. (Almería, 3 de junio de 1972). 
Aguas abajo en la margen derecha de la rambla de Guazamara, la población contaba con las aguas del Pilar de la Ermita Vieja. Disponía de abrevadero, lavadero y balsa de acumulación de las aguas, que posteriormente se distribuían para riego en esa margen derecha. Y en la margen izquierda, paraje del Rincón, el nacimiento de la Fuente del Vinagre, con balsa y para riego en esa margen.

En el año 2014, en Pulpí y áreas colindantes de Huércal Overa

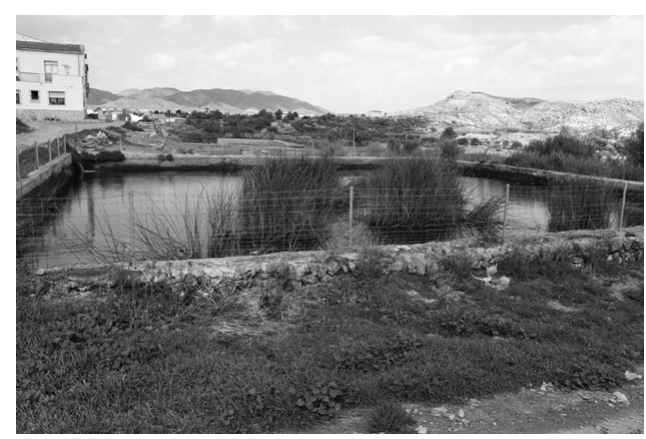

Figura 16. Balsa que recibe las aguas del Pilar de la Ermita Vieja, en la margen derecha de la rambla de Guazamara Cuevas del Almanzora).

Fuente: Gómez Espín, J.M. a 04/04/2015.

y Cuevas del Almanzora, llegamos

a inventariar 62 galerías. (Gil, E.; García, P.J.; Gómez, J.M.a; Almela, R. 2014: 19-21). En parajes como Las Canalejas, continuaban funcionales la cimbra de La Mucha, la de la Balsa Redonda, y la de Las Agüicas. Y en Los Vizcainos estaban dando agua los dos qanates de la Balsa del Negro, la cimbra del Balsón, los dos qanates de Miguel Parra, y los del Pozo Vizcaino y el Romeral.

\section{CONCLUSIONES Y PROPUESTAS}

La Junta Central de Usuarios de Aguas del Valle del Almanzora, en los últimos años, ha llevado a cabo un estudio agronómico, sobre los consumos de agua de las principales masas de cultivo con riego localizado por«goteo» en la Comarca, especialmente en el Bajo Almanzora.

Para una superficie regable de 36.000 hectáreas, con la participación de las distintas masas de cultivo en riego localizado (Tabla 4), se evaluaban las necesidades en 126 hectómetros cúbicos.

Tabla 4. Necesidades de agua, según masas de cultivo con riego localizado, en la Comarca del Valle del Almanzora

\begin{tabular}{|l|c|c|c|}
\hline \multicolumn{1}{|c|}{ Masa de cultivo. } & Superficie (ha). & $\begin{array}{c}\text { Volúmenes medios } \\
\left(\mathbf{m}^{\mathbf{3}} \mathbf{/ h a} / \mathbf{a n ̃ o}\right) .\end{array}$ & Volumen anual $\left(\mathbf{m}^{\mathbf{3}} \mathbf{) .}\right.$ \\
\hline Hortícolas & 25.200 & 2.091 & 52.682 .965 \\
\hline Cítricos & 9.000 & 7.226 & 65.035 .843 \\
\hline Resto arbolado & 1.800 & 4.625 & 8.325 .394 \\
\hline TOTALES & 36.000 & 3.501 & 126.044 .202 \\
\hline
\end{tabular}

Fuente: Elaboración propia. Junta Central de Usuarios de Aguas del Valle del Almanzora. Estudio Agronómico 2003-2014. 


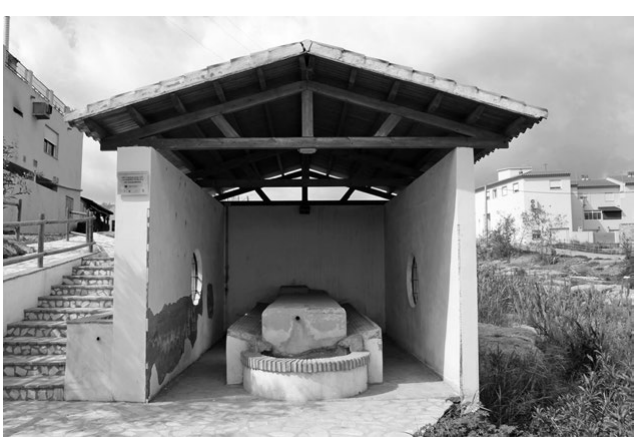

Figura 17. Lavadero rehabilitado que recibía las aguas del Pilar de la Ermita Vieja, en la margen derecha de la rambla de Guazamara (Cuevas de Almanzora). Fuente: Gómez Espín, J.M. ${ }^{a}$ 04/04/2015.

En cuanto a recursos de agua para atender esas demandas (Tabla 5), las aguas alumbradas por las galerías (manantiales del Alto y Medio Almanzora) representaban algo más del 10 por ciento $(11,11$ \%). La expansión del las áreas regadas en la zona Baja del Almanzora (30.873 hectáreas), se ha realizado, sobre todo, con recursos foráneos (Trasvase Tajo-Segura y Conexión Negratín-Almanzora) que aportarían casi la mitad del total de los volúmenes de agua para riego $(43,65 \%)$.

Tabla 5. Recursos de agua para el Valle del Almanzora en el año hidrológico 2014/2015

\begin{tabular}{|c|c|c|}
\hline Origen del agua & $\begin{array}{l}\text { Volúmenes } \\
\left(\mathbf{H m}^{3} / \mathbf{a n ̃ o}\right)\end{array}$ & $\begin{array}{c}\text { Volúmenes en } \\
(\%) .\end{array}$ \\
\hline Trasvase Tajo-Segura & 12 & 9,53 \\
\hline Conexión Negratín-Almanzora & 43 & 34,13 \\
\hline Embalse de Cuevas de Almanzora & 22 & 17,46 \\
\hline Manantiales Alto y Medio Almanzora & 14 & 11,11 \\
\hline Pozos Alto y Medio Almanzora & 9 & 7,14 \\
\hline Pozos salobres Bajo Almanzora & 6 & 4,76 \\
\hline Desalación & 20 & 15,87 \\
\hline TOTALES & 126 & 100,00 \\
\hline
\end{tabular}

Fuente: Elaboración propia. Aguas de Almanzora, S.A.

Este balance pone de manifiesto que los aprovechamientos tradicionales fueron capaces de crear un rosario de huertas y asistir a los cultivos del secano tradicional. En un aprovechamiento integral, dónde esas aguas para riego dieron también servicio de abastecimiento a las poblaciones, a los ganados, y la energía hidráulica para mover las piedras de molinos de cubo y almazaras. Pero ni aún con las modernas técnicas de riego localizado, podrían suministrar los volúmenes de agua que necesitan las nuevas áreas regadas, cuyas producciones han hecho posible detener la emigración tradicional.

A la aportación de las aguas alumbradas, como recursos propios, en un territorio de escasez como el Sureste., hemos de sumar el valor del patrimonio 


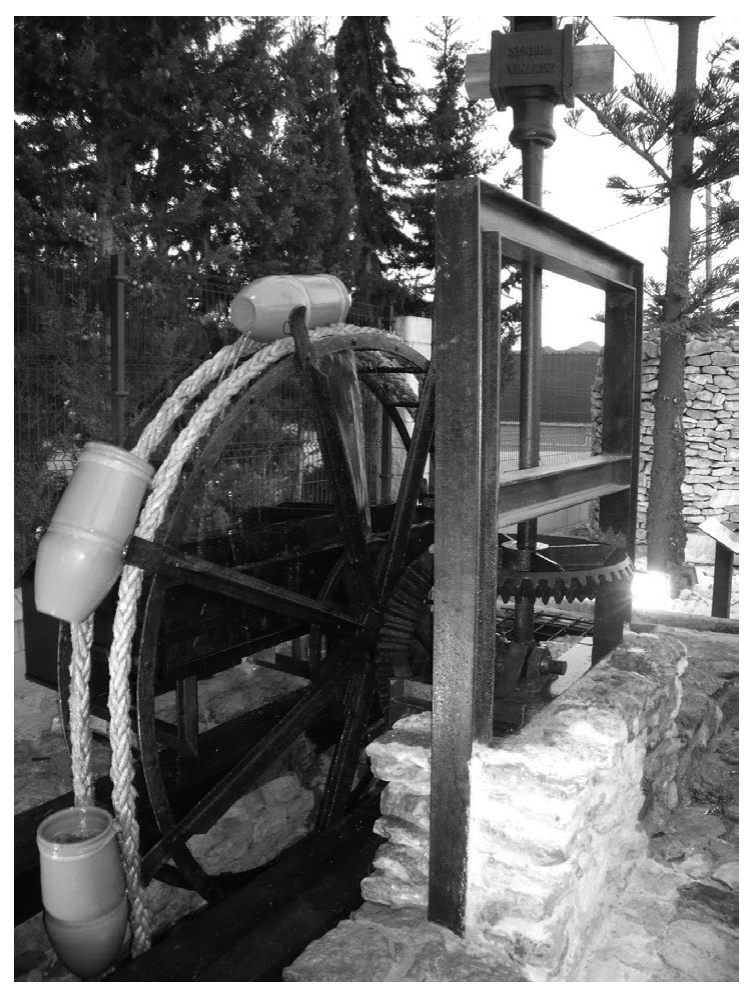

Figura 18. Reproducción de una noria de tiro o de sangre en el Museo del Agua, en el El Saltador (Huércal-Overa). Fuente: Gómez, J.M. ${ }^{a}$ y Gil, E. 28/06/2014.

hidráulico que encierran galerías, lumbreras, bocaminas, acueductos, balsas, abrevaderos, lavaderos, molinos hidráulicos de cubo, almazaras y otras fábricas hidráulicas, etc. Rehabilitadas para ponerlas en valor junto a los entornos dónde se encuentran, pueden ser, a nivel local, incentivos para el desarrollo. Centros de interpretación de los usos del agua en los medios semiáridos, $\mathrm{y}$ «sendas del agua» en estos paisajes para actividades lúdico-recreativas y científico-culturales, etc.

\section{FUENTES Y BIBLIOGRAFÍA}

Aliaga, I.; Gil, E.; Gómez, J.M.; LóPez, J.A.; Martínez, R. (2007): Sistemas Locales de Recursos Propios de Agua en la Región de Murcia: Minados y Galerías. Ente Público del Agua. Universidad de Murcia. Murcia 190 pp.

Fernández Bolea, E. (2006): Agua y vida en Cuevas de Almanzora. Una historia de luchas y anhelos (siglos XVI-XXI). Arráez Editores, S.L., Mojacar (Almería). 334 pp. Fernández Bolea, E. (2011): Memoria del Agua. De los riegos tradicionales a la Sociedad Agraria de Transformación de Los Guiraos (50 años de luchas y logros). Arráez Editores, S.L. Mojacar (Almería). 222 pp. 
Ferre Bueno, E. (1979): El Valle del Almanzora. Estudio Geográfico. Excma. Diputación Provincial. Caja Rural Provincial. Monte de Piedad y Caja de Ahorros de Almería. Almería. 494 pp.

Garcia AsEnsio, (1908-1909): Historia de la Villa de Huércal-Overa y su comarca. Volumen I. Tipografía de José Antonio Jiménez. Murcia. 542 pp.

Garcia Asensio, (1910): Historia de la Villa de Huércal-Overa y su comarca. Tipografía de José Antonio Jiménez. Murcia. Volumen II, 688 pp. Volumen III, 621 pp.

Gil Meseguer, E. (2014):«Aprovechamiento y gestión del agua en medio semiáridos». PhICARIa II Encuentros Internacionales del Mediterráneo. Uso y gestión de recursos naturales en medios semiáridos de ámbito mediterráneo. Univ. Popular de Mazarrón. Pp.39-52.

Gil Meseguer, E.; Gómez Espín, J. M. ${ }^{a}$ (2012): «La investigación en España sobre los sistemas de captación y conducción de pozo horizontal (galería), asociada o no a presa subálvea». Patrimonio hidráulico y cultura del agua en el Mediterráneo. Fundación Séneca. AECID. Campus de Excelencia Internacional «Mare Nostrum». Murcia. Pp.189-202.

Gil Meseguer, E.: Gómez Espín, J.M. (2014): «Las aguas de fuentes en Huércal-Overa (Almería). El modelo de la Sociedad Colectiva de aguas titulada $<$ La Asunción $>$ ». Libro jubilar en homenaje al Profesor Antonio Gil Olcina. Universidad de Alicante. Pp. 215-230.

Gil Meseguer, E.: Gómez Espín, J.M. (2015): «Cambios en la ordenación territorial del Bajo Almanzora auspiciados por los trasvase Tajo-Segura y Negratín-Almanzora». De la Riva, J.; Ibarra, P.; Montorio, R.; Rodríguez, M. (Eds.). Análisis espacial y representación geográfica: innovación y aplicación. Zaragoza. Pp. 139-147.

Gil Meseguer, E.; Martinez Medina, R.; Gómez Espín, J.M. ${ }^{a}$ (2011): «Modelos de uso sostenible del agua: las galerías asociadas a presa subálvea». Scripta Nova. Revista Electrónica de Geografía y Ciencias Sociales. Volumen XV, n. ${ }^{\circ} 374.10$ de septiembre de 2011. Barcelona. Pp. 1-16.

Gil Meseguer, E.; García SÁnchez, R.M.; Gómez Espín, J.M. a (2013): «Funcionalidad de las técnicas del pozo horizontal (galería) para la captación y conducción de aguas en el Sureste de España». Las galerías de agua en la Región Noroccidental de Túnez. Patrimonio hidráulico Mediterráneo. Agencia Española de Cooperación Internacional para el Desarrollo. Universidad de Valencia. Pp.147-158.

Gil Meseguer, E.; García Martinez, P.J.; Gómez Espín, J.M.a; Almela Pérez, R. (2014): El dinamismo del regadío de Pulpí. Comunidad de Regantes de Pulpí. Murcia. 222 pp.

Gil Meseguer, E.; López Fernández, J.A.; Gómez Espín, J: M. a (2015): «Regadíos de turbias y secanos asistidos en el Sureste de España. El sistema del Azud de Guadalupe o presa de derivación de avenidas de la Rambla del Zoco». Revista Murciana de Antropología, n. ${ }^{\circ}$ 22. (Número monográfico de Cultura de Agua en Murcia y Sureste Español). Universidad de Murcia. Pp. 161-176.

Gil OlcinA, A. (1993): La propiedad de las aguas perennes en el Sureste ibérico. Servicio de Publicaciones. Universidad de Alicante. $191 \mathrm{pp}$.

Goblot, H. (1979): Les QANATS. Une Technique d'acquisition de l'eau. Mouton Editeur. París - La Haye - New York. 236 pp. 
Gómez Espín, J. M. (2004): Aprovechamiento integral del agua de la Rambla de Nogalte (Puerto lumbreras-Murcia). CAM Proyecto Agua. Ayuntamiento de Puerto Lumbreras. Universidad de Murcia. Murcia. 190 pp.

Gómez Espín, J. M. a; Gil Meseguer, E.; Aliaga Sola, I.; López Fernández, J. A.; Martínez MedinA, R.; (2007): «Las galerías, construcciones para alumbrar agua de freáticos próximos en el NE de la Región de Murcia: minados con espejuelos en Jumilla». Revista Investigaciones Geográficas, n. ${ }^{\circ}$ 42. Universidad de Alicante. Pp. 89-107.

Hermosilla Plá, J. et al. (2004): «Las galerías drenantes de la provincia de Almería. Análisis, y clasificación tipológica». Cuadernos de Geografía, $n .^{\circ} 76$. Universidad de Valencia. Págs. 125-154.

Hermosilla Plá, J. (Director) (2006): Las galerías drenantes del Sureste de la Península Ibérica. Ministerio de Medio Ambiente. Madrid. 227 pp.

Hermosilla Plá, J. (Director) (2008): Las galerías drenantes en España. Análisis y selección de qanat(s). Ministerio de Medio Ambiente, y Medio Rural y Marino. Madrid. 269 pp.

Hermosilla Plá, J. (Director) (2010): Los nuevos regadios históricos españoles. Paisajes culturales, paisajes sostenibles. Ministerio de Medio Ambiente y Medio Rural y Marino. Madrid. 608 pp.

Junta Central de Usuarios de Aguas del Valle del Almanzora (2001): Inventario de comunidades de regantes de la Comarca del Almanzora. Octubre, 2001. Sin Paginar.

Junta de Andalucía. (2010): Manantiales y fuentes de Andalucía. Hacia una estrategia de Conservación. Universidad de Granada. Junta de Andalucía. (http:/www. conocetusfuentes.com).

Llobet ReVERTER, S. (1958): «Utilización del suelo y economía del agua en la región semiárida de de Huércal-Overa (Almería)». Revista Estudios Geográficos, n. ${ }^{\circ} 70$. Febrero 1958. Madrid. Pp. 5-21.

López Fernández, J.A.; Gil Meseguer, E.; Gómez Espín, J: M. a (2015): «Proyectos para aumentar el caudal en el nacimiento del río Mula a través de la técnica del pozo horizontal». Revista Murciana de Antropología, n. ${ }^{\circ} 22$. (Número monográfico de Cultura de Agua en Murcia y Sureste Español).Universidad de Murcia. Pp. 137-160.

Morales, A.; Box, M.; Marco, J.A. (1989): «El aprovechamiento de las aguas de avenida: derecho consuetudinario y disposiciones legales». Avenidas fluviales $e$ inundaciones en la Cuenca del Mediterráneo. Instituto Universitario de Geografía. Universidad de Alicante. CAM. Murcia. Pp.553-563.

Morales, A.; Box, M.; Marco, J.A. (1991): «El medio físico y la presa del Román (Jumilla): un emplazamiento favorable». Investigaciones Geográficas, n. ${ }^{\circ} 9$. Universidad de Alicante. Murcia. Pp.69-80. 